\title{
Near Real Time Applications for Maritime Situational Awareness
}

\author{
E. Schwarz *, D. Krause, M. Berg, H. Daedelow, H. Maass \\ German Aerospace Center (DLR), Earth Observation Center, German Remote Sensing Data Center (DFD), National Ground
} Segment, Kalkhorstweg 53, 17235 Neustrelitz, Germany - Egbert.Schwarz@dlr.de

KEY WORDS: TerraSAR-X, near real time, ship, oil, wind, wave

\begin{abstract}
:
Applications to derive maritime value added products like oil spill and ship detection based on remote sensing SAR image data are being developed and integrated at the Ground Station Neustrelitz, part of the German Remote Sensing Data Center. Products of meteo-marine parameters like wind and wave will complement the product portfolio. Research and development aim at the implementation of highly automated services for operational use. SAR images are being used because of the possibility to provide maritime products with high spatial resolution over wide swaths and under all weather conditions. In combination with other information like Automatic Identification System (AIS) data fusion products are available to support the Maritime Situational Awareness.
\end{abstract}

\section{INTRODUCTION}

\subsection{Objective}

Marine resources become more and more important for the European and global economy. Furthermore the progression of maritime economic activities is based on a safe and secure environment. The priorities for the EU Integrated Maritime Policy, created in 2007, are to improve maritime safety and security, reduce pollution and to fight against illegal activities. Its primary goal is to achieve good environmental status (GES) for EU marine waters by 2020. (COM, 2012)

In Germany DLR and industrial partners launched in 2012 a major multi-annual research and development programme in "Maritime Safety and Security". The main priorities for the development and operation of Integrated Maritime Services are the improvement of maritime safety and security. Remote sensing provides outstanding capabilities to monitor large coverages in a minimum time frame.

Research and development aims at automated near real time (NRT) applications based on a remote sensing multi-sensor approach in a synergetic use with ground-based sensor data. This enables supporting of service providers and Copernicus downstream services, supplying timely information for the maritime domain.

\subsection{Background}

The German Remote Sensing Data Center participates in several maritime projects. Three of these projects with DLR involvement are the European Space Agency (ESA) project European "Maritime Security" Services (MARISS) and the EU FP7 projects "Development of Pre-operational Services for Highly Innovative Maritime Surveillance Capabilities" (DOLPHIN) and "Support to Pre-Operational Validation of the High-Time Critical Components of CONOPS” (SAGRES). The European Maritime Security Service, MARISS was the first phase of a pan-European activity of ESA under Copernicus, formerly known as "Global Monitoring for Environment and Security" (GMES).
The department National Ground Segment of DFD, situated in Neustrelitz, in the federal state of Mecklenburg - Western Pomerania, handles the reception, processing, distribution and long term archiving of payload data for a multitude of satellite missions. The work is carried out as part of German space program or on behalf of private industry, and in cooperation with international space agencies.

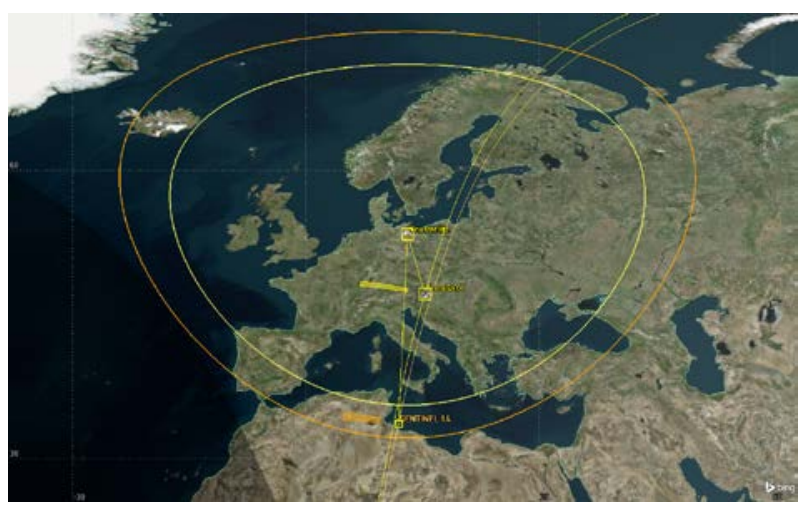

Figure 1. Ground Station Neustrelitz, acquisition circle for Sentinel-1 and TerraSAR-X; 5 degree elevation

As Figure 1 shows, the acquisition circle of Neustrelitz (approximately by elevation of five degree) allows coverage from the North Atlantic to the Mediterranean Sea. The range of activity was continuously expanded and further developed over more than 45 years of ground station operations. Mastery of the entire value adding chain from data reception to data processing and final distribution ensures that users can obtain high quality homogenized data products which are free of systematic artefacts and of high information content. The relevant experience covers all parts of the service cycle from interface definition and implementation, to near real time data reception and operational near real time processing, product generation, analysis and dissemination to users in the maritime domain. 


\section{DATA AND METHODS}

\subsection{SAR Data}

Satellite data received directly in Neustrelitz from several Synthetic Aperture Radar (SAR) missions serve as primary input data for deriving value-added information products. In the past, data from the ERS-2 and Envisat satellites were used. Currently, due to image resolution, data from TerraSAR-X and TanDEM-X are preferentially processed. Both satellites acquire high quality radar images using active phased array X-band Synthetic Aperture Radar (SAR) antenna. The SAR instrument works independent of day time and weather conditions and is therefore eminently suitable for target detection, oil spill detection as well as applications to derive wind and sea state information. Within the SAGRES project ship detection capabilities were expanded to include CosmoSkyMed and Radarsat-2 data. In 2015 the development of the collaborative ground segment for the Copernicus Mission Sentinel-1 should lead to further improvement in the spatial and temporal availability of data from Neustrelitz. Furthermore the ground station will be extended by a Radarsat- 2 Regional Ground System (RGS) enabling real time access to Radarsat-2 data for scientific purposes.

\subsection{Environment}

To fulfil user requirements and achieve relevant processing times of less than 15 minutes after image acquisition, the efficient use of the processing hardware as well as the optimisation of transfer time is mandatory. With respect to the processing framework architecture parallel processing is only supported for shared file systems. In the TerraSAR-X ground segment the Quick File System (QFS) is used to provide such functionality to all processing nodes. The storage behind this shared file system is built on solid-state disks (SSDs), connected via fibre channel, to support fast data processing.

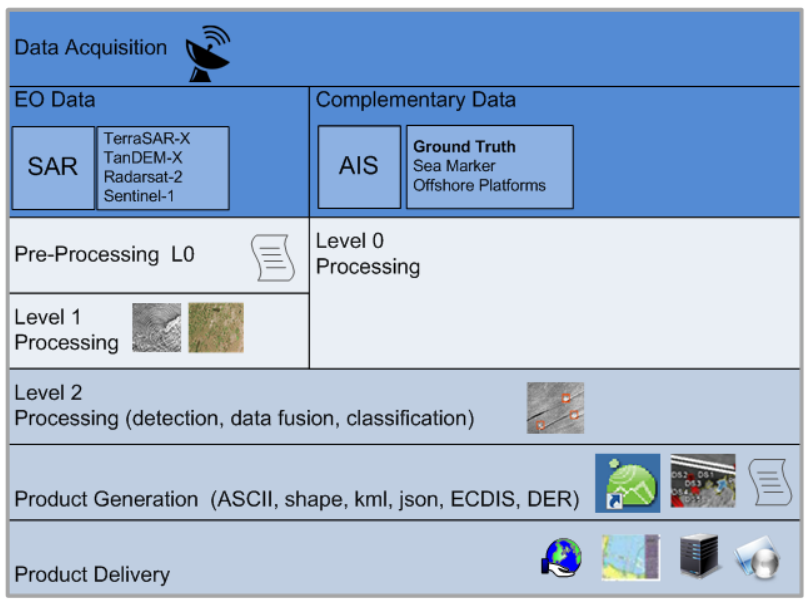

Figure 2. Workflow to supply satellite-based maritime information products (current status)

The overall processing is running fully automated. SAR images downlinked to the ground station are transferred with approximately 10 seconds delay directly to the processing pickup point which is part of a Storage Area Network (SAN). The processing system is divided into two sub chains: "NRT" and "Standard", where Standard means processing of non-time critical Level 1 catalogue and future product orders. This approach guarantees the availability of processing resources for NRT processing at any time. All processing control functions are implemented within the Processing System Management (PSM), part of the Data Information Management System (DIMS) (Wolfmüller et al., 2008). DIMS is a work flow management system which contains also the order interface needed for order submission, and production control for processing request generation. The PSM covers common functions of processing systems and supports different processing scenarios. For example the PSM provides raw data ingestion, request queuing, processing workflow scheduling, production and data dissemination. The PSM and all processing requests are monitored and controlled with DIMS Operating Tool (OT) which provides a set of views and allows operator interactions. Figure 2 shows all steps from data acquisition to product dissemination.

\subsection{NRT Application}

The mission TerraSAR-X is currently the main driver for maritime NRT development. Figure 3 shows the principle workflow for NRT products including ship detection, one of the first value adding products, implemented in the TerraSAR-X ground segment.

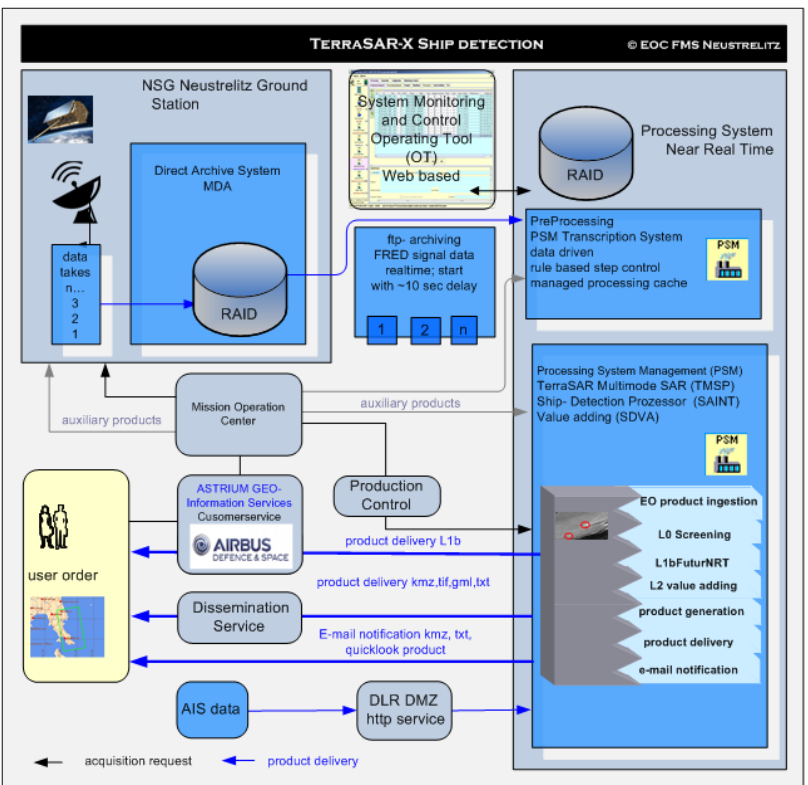

Figure 3. TerraSAR-X NRT ship detection product order and delivery chain

The ship detection order option is available for NRT future product orders and can be requested in addition to the standard Level 1b Multilook Ground Range Detected (MGD) product. Data reception and NRT processing are carried out at the DLR Neustrelitz Ground Station. Screening (Level 0) and preprocessing (Level 1b) of SAR data are tasks of the TerraSAR Multi Mode SAR Processor (TMSP) (Breit et al, 2008). The processing is performed on data take level. Each data take represents one user order and contains the encrypted raw data of an image acquisition taken in one of the supported imaging modes. For fast data processing PSM rules are implemented to fetch NRT flagged data takes first. After data decryption the data take is handed over to the NRT processing system where the data are mapped directly to the already scheduled processing request. In case of NRT future product orders, the processing 
starts immediately as the predicted orbit and predicted attitude auxiliary product can be used. For NRT value adding orders the TMSP configuration allows scene slicing in dependency of available processing configuration. As a result the whole scene is divided in several sub-scenes with small overlaps after the L0 screening. Afterwards all sub-scenes are being processed in parallel on different processing nodes. This improves the performance not only of processing, but also concerning the product delivery. For value adding processing (Level 2) the following processors are currently integrated:

1. Oil spill classification processor (Singha et al., 2014)

2. SAR AIS Integrated Toolbox (SAINT)

for ship detection. (Brusch et al., 2011)

3. XMOD-2 to derive wind speed and direction. (Jacobsen et al., 2013)

4. XWAVE to derive wave height and wave length (Pleskachevsky et al., 2015)

All core processor modules used for value adding are developed at the Maritime Safety and Security Lab in Bremen, part of DLR's Remote Sensing Technology Institute.

\section{RESULTS}

\subsection{Oil Spill Detection}

In 2012 European Maritime Safety Agency (EMSA) and DLR launched the project "Monitoring of off shore platforms using TerraSAR-X Imagery". The scope was to examine how TerraSAR images and enhanced oil spill analysis can support the EMSA operated satellite based oil spill monitoring and vessel detection services. Supported by the national project "Real-time Services for Maritime Security" (EMS) a prototype application for semi-automated oil spill detection was developed. The application supports stand-alone processing based on the Level $1 \mathrm{~b}$ product as well as integration in the already existing NRT workflow. All processing steps are triggered by the oil detection processing rule, added to the PSM control system which is calling a sequence of additional processing steps. Main element of the workflow is the classification processor, based on a trained neural network.

Virtual Network Computing (VNC) is implemented to enable remote operator interaction. The interaction is needed to select a region of interest (ROI) containing an oil spill or 'look-alike's candidates. Multiple choice is supported. Only the selected ROI's are the input for the classification processor. As a result from the classification possible oil spills are highlighted. The processor result has to be confirmed by the operator before additional processors for product generation and dissemination are executed.

After product generation different product formats are available in addition to the NRT L1b product delivery via SFTP, to support fast delivery via e-mail (e.g., kmz, pdf, shape- and text files), dissemination via Web Mapping Server (e.g. tiff and ESRI shape file layer) as well as formats compliant to the EMSA CSN Standard. Figure 3 shows the pdf example which can be attached to an e-mail notification, to inform the user that the product was delivered.

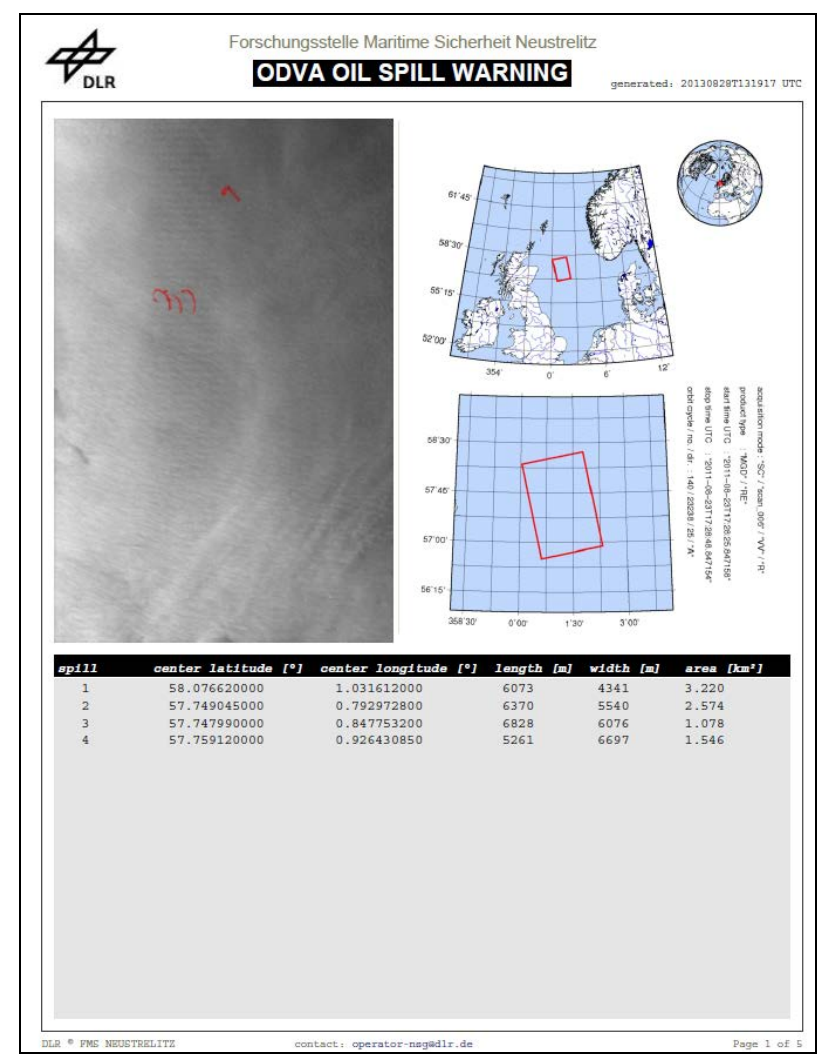

Figure 3. pdf product example

\subsection{Ship Detection}

Output of the ship detection processing chain are the value added products in different formats in addition to the standard L1b product as specified within the ground segment. The generated products contain information such as the position, length, width and heading of the target. The detection result is highly depending on the direction of the vessel, the SAR incidence angle and the sea state. For this reason quality information is attached for each target representing the confidence level for detection. Ground truth information like terrestrial AIS data for the North- and Baltic Sea are acquired via network also in real time and merged to the SAR based intermediate product during post processing before generating the final output.

The fusion with AIS data from terrestrial and satellite sources creates a more significant picture with additional information e.g. ship name, ship class and destination. AIS messages are broadcasted and mandatory for all vessels larger than 300 GRT. Currently automatic-correlation of SAR-detections with AISmessages is done on basis of the shortest distance. The method will be further developed and more sophisticated approaches will be integrated in the future.

The product dissemination method is as described in the previous chapter. The L1b image products are delivered independently from the value adding results. Products such as quicklook images, ASCII files with ship position and a kmz file for displaying results in Google Earth are delivered via automatically generated e-mail (see figure 4). 


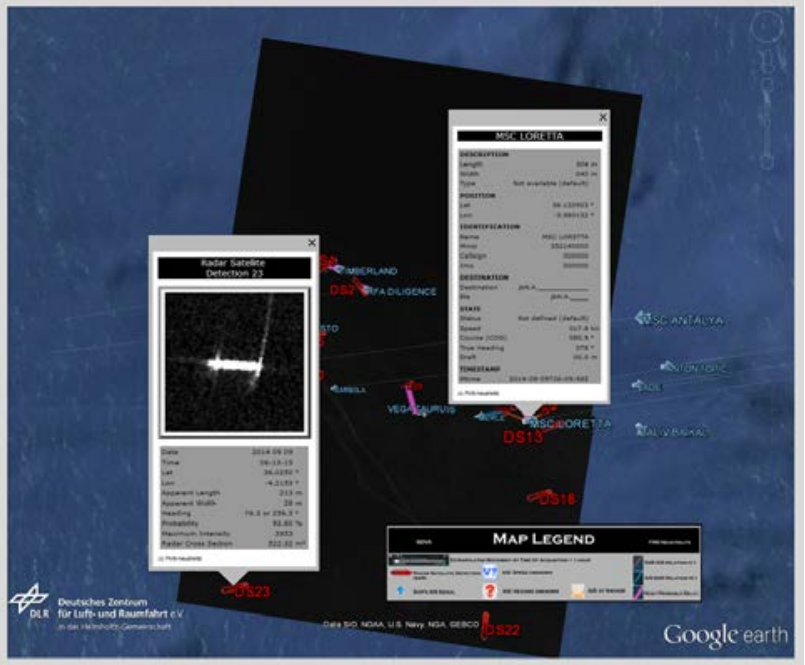

Figure 4. kmz output product extract; image data product TerraSAR-X merged with ground based AIS data;

Google Earth used for product illustration

The kmz file contains a map legend and separate product layer. The layer information for "MOST PROBABLE RELATIONS" is highlighting the most probable relation between SAR detection and the corresponding AIS message. The information is also available in the embedded in plain ASCII text file. For each detected ship a "Radar Satellite Detection" pop-up window is embedded. This window contains the ship detection information and a thumbnail (gif) showing the detected object. AIS pop-up windows are embedded as well if the data was available in real-time for the ROI. For NRT delivery some rules have to be applied to be compliant with German Law. For this reason the thumbnail spatial resolution is resampled to worse than 10 meter. In addition products like the medium resolution geotif file, json files, EMSA DER package and ESRI shape files for the different layers are available for dissemination via delivery- and Web GIS server.

\subsection{SAR Wind Products}

The automatically generated SAR wind field information is available from the Level 2 wind processing chain. Core function is the XMOD-2 algorithm of the wind processor for X-Band data. For C-Band data the CMOD-5 approach is implemented. Both algorithms derive wind fields from SAR images of sea surfaces by means of empirical geophysical model functions (GMF) with specifically adjusted coefficients. The processor will be integrated as a second value adding step in parallel to the ship detection. An autonomous processing rule is called by the PSM control system based on the request parameter. The Figure 5 shows the level 2 workflow elements, part of the entire processing chain.

The fully automated derivation of wind direction from wind streaks on the ocean surface is currently under development. Therefore in the meantime the wind field extraction needs estimated wind direction in addition to the SAR L1b image input. This input is generated by the Weather Research and Forecasting (WRF) Model (Michalakes et al., 2004). Figure 5 shows the principle approach, running a single PSM application of the WRF model to provide the information, not only for TerraSAR-X processing. The WRF model is chosen because it can handle all popular weather models as input and can also add observation and calibration data. Due to the limited ROI, the calculation time, needed to run the WRF model, is minimal and results are generally available after L1b processing completion. As a result the netCDF output is generated, containing the wind direction and intensity (WD10). Based on the product generation approach also implemented for oil and ship detection different products e.g. netCDF and $\mathrm{kmz}$, as well as ESRI shape file layers for dissemination via delivery server or Web Mapping Server are available.

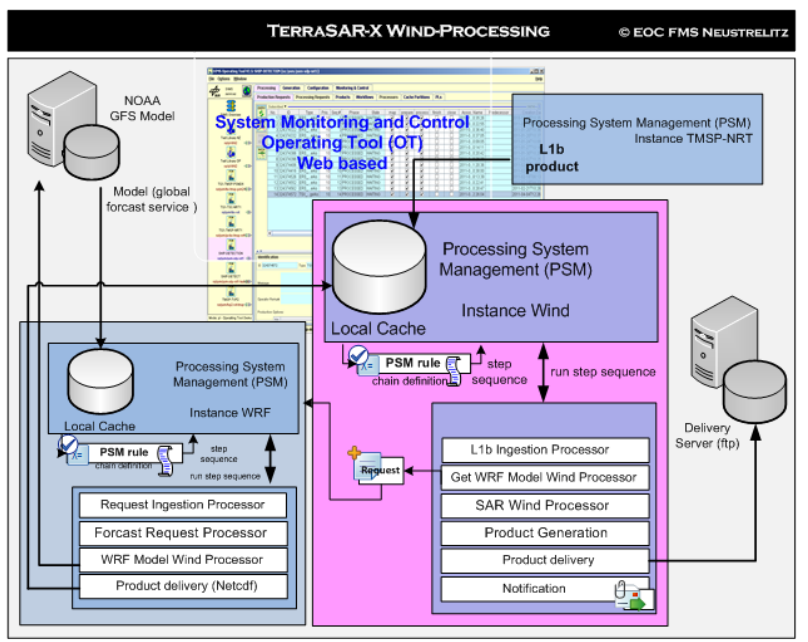

Figure 5. TerraSAR-X NRT wind processing work flow

\subsection{SAR Wave Products}

Current research at the Maritime Safety and Security Lab in Bremen investigates the estimation of sea state conditions from SAR images. For X-Band data the XWAVE and for C-Band data the CWAVE algorithm is applied. Both algorithms analyse the modulation of backscatter intensities in a sub-scene using the Fast Fourier Transformation (FFT) and output significant wave height, peak period and peak wave length of sea state. The parameters of the XWAVE and CWAVE algorithms have been adjusted based on collocated measurement data of NOAAbuoys in the open ocean and validated with an independent buoy dataset as well as model data from the German Meteorological Service (DWD) (Pleskachevsky et al., 2015). The processor requires wind speeds higher than 2 meters per seconds (otherwise sea state cannot be derived) and resolution better than 10 meters (e.g. TerraSAR-X Stripmap), ScanSAR with 18 meters resolution can only be used for waves longer than 100 meters in open sea.

The processing chain is currently implemented in a single solution, running the Level 2 wave detection in a stand-alone PSM environment. Later on the whole processing rule will be integrated in parallel to the wind detection. As figured out for the wind detection in chapter 3.3 an autonomous processing rule is called by the PSM control system based on the specified request parameter. Following the product generation approach implemented for wind detection the same product types will be available for dissemination via delivery server or Web GIS server. One product example (grid) derived from TerraSAR-X Stripmap and visualised in ArcMap is shown in Figure 6. The SAR image is overlaid by the wave product (significant wave height only). Interpolation is done for the values between the results generated by the processor (dots). 


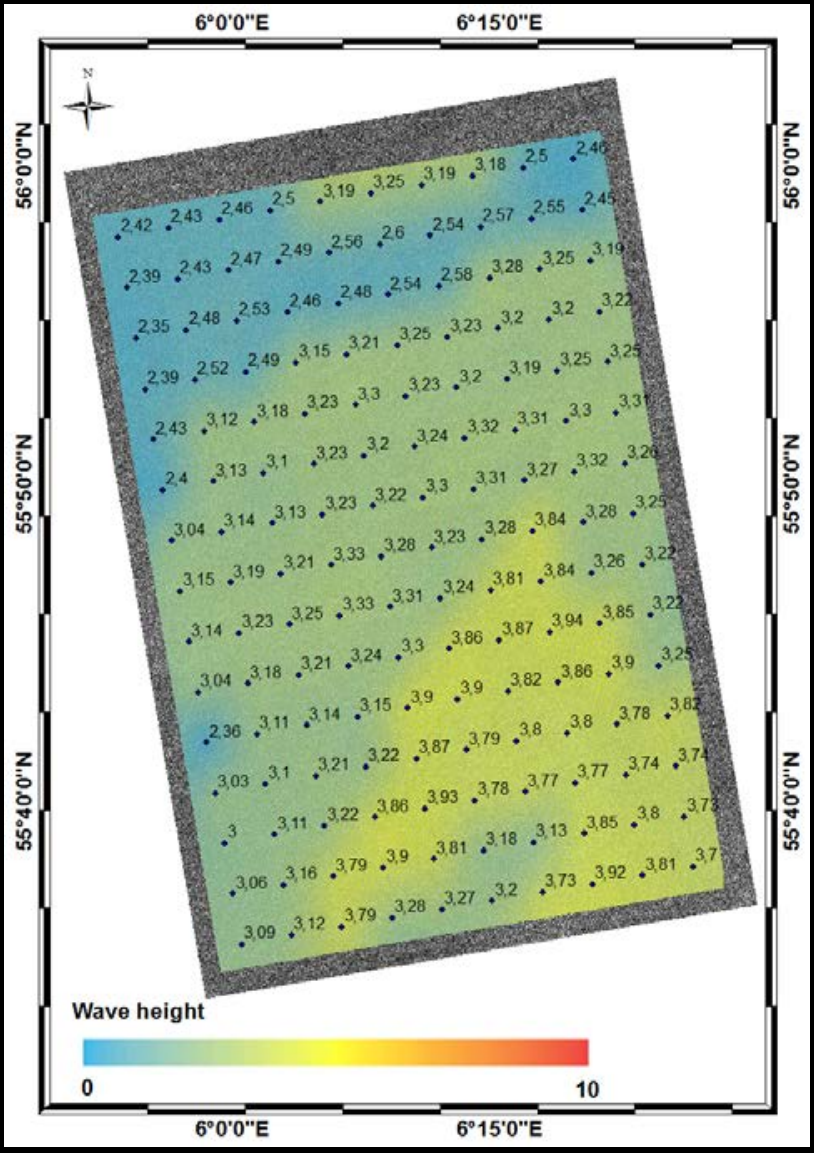

Figure 6. TerraSAR-X wave product example

\subsection{Outlook}

The Ground Station Neustrelitz will be upgraded during 2015 to enable Radarsat-2 real-time reception and processing for science purposes. Furthermore the processing capabilities will be extended to support Sentinel-1 data reception in pass through mode (direct downlink) in frame of the Copernicus Collaborative Ground Segment. The whole post processing chain will be developed and implemented, extended by value adding processing capabilities to generate higher value maritime products from Sentinel-1 data.

\section{CONCLUSION}

SAR images are more and more in use to support maritime surveillance. The algorithms for value adding services in the maritime domain are available for operational use. Near real time capabilities are amongst others the main requirements for such services. New satellite systems will increase the availability of image data, but further research and development are needed to support new image modes, e.g. Sentinel-1 TOPS mode. The integration of further data streams, not only from radar but also optical sensors, as well as satellite based AIS will increase the reliability of the service.

\section{REFERENCES}

COM(2012) 491 final. Progress of the EU's Integrated Maritime Policy, Report from the Commission to the European Parliament, the Council, the European Economic and Social Committee and the Committee of the Regions.
Breit, H., Schättler, B., Fritz, T., Balss, U., Damerow , H., and Schwarz, E., 2008. TerraSAR-X Payload Data Processing: Results from Commissioning and Early Operational Phase. In: Proceedings of the IEEE Geoscience and Remote Sensing Symposium (IGARSS), Boston, USA, pp. 209-212.

Brusch, S., Lehner, S., Fritz, T., Soccorsi, M., Soloviev, A., and van Schie, B., 2011. Ship Surveillance With TerraSAR-X. IEEE Transactions on Geoscience and Remote Sensing, 49(3), pp. 1092-1103

Jacobsen, S., Lehner, S., Pleskachevsky, A., and Rosenthal, W., 2013. Meteo-marine parameters and their variability observed by high resolution satellite radar. In: Proc. 'Living Planet Symposium 2013', Edinburgh, UK, 9-13 September 2013 (ESA SP-722, December 2013). ISBN 978929221286 5. ISSN 1609-042X.

Michalakes, J., Dudhia, J., Gill, D., Henderson, T., Klemp, J. Skamarock, W., and Wang, W., 2004. The Weather Reseach and Forecast Model: Software Architecture and Performance. In: Proceedings of the 11th ECMWF Workshop on the Use of High Performance Computing In Meteorology, 25-29 October 2004, Reading, U.K.

Pleskachevsky, A., Gebhardt, C., Rosenthal, W., Lehner, S., Hoffmann, P., Kieser, J., Bruns, T., Lindenthal, A., Jansen, F., and Behrens, A., 2015. Satellite-Based Radar Measurements for Validation of High-resolution Sea State Forecast Models in the German Bight, In: Proc. ISRSE36, The 36th International Symposium on Remote Sensing of Environment, 11 - 15 May 2015, Berlin, Germany.

Singha, S., Velotto, D., and Lehner, S., 2014. Near real time monitoring of platform sourced pollution using TerraSAR-X over the North Sea. Marine Pollution Bulletin, 86(1-2), pp. 379-390. Elsevier Ltd. DOI: 10.1016/j.marpolbul.2014.06.041. ISSN 0025-326X.

Wolfmüller, M., Dietrich, D., Sireteanu, E., Kiemle, S., Mikusch, E., and Böttcher, M., 2008. Dataflow and Workflow Organization - The Data Management for the TerraSAR-X Payload Ground Segment. IEEE Transactions on Geoscience and Remote Sensing, 47(1), pp. 44-50 